\title{
Repair of Specific Base Pair Mismatches Formed during Meiotic Recombination in the Yeast Saccharomyces cerevisiae
}

\author{
PETER DETLOFF, ${ }^{1,2}$ JENNIFER SIEBER, ${ }^{1}$ AND THOMAS D. PETES ${ }^{1 *}$ \\ Department of Biology, University of North Carolina, Chapel Hill, North Carolina 27599-3280, ${ }^{1}$ and Department of \\ Molecular Genetics and Cell Biology, University of Chicago, Chicago, Illinois $60637^{2}$
}

Received 20 August 1990/Accepted 9 November 1990

\begin{abstract}
Heteroduplexes formed between DNA strands derived from different homologous chromosomes are an intermediate in meiotic crossing over in the yeast Saccharomyces cerevisiae and other eucaryotes. A heteroduplex formed between wild-type and mutant genes will contain a base pair mismatch; failure to repair this mismatch will lead to postmeiotic segregation (PMS). By analyzing the frequency of PMS for various mutant alleles in the yeast $\mathrm{HIS} 4$ gene, we showed that $\mathrm{C} / \mathrm{C}$ mismatches were ineficiently repaired relative to all other point mismatches. These other mismatches (G/G, G/A, T/T, A/A, T/C, C/A, A/A, and T/G) were repaired with approximately the same efficiency. We found that in spores with unrepaired mismatches in heteroduplexes, the nontranscribed strand of the HIS4 gene was more frequently donated than the transcribed strand. In addition, the direction of repair for certain mismatches was nonrandom.
\end{abstract}

When a diploid strain of Saccharomyces cerevisiae undergoes meiosis, heterozygous alleles $A$ and $a$ usually segregate $2 A: 2 a$ into the four spores of the ascus. Two types of aberrant segregation are observed: gene conversion events ( $3 A: 1 a$ or $1 A: 3 a$ segregation) and postmeiotic segregation (PMS) events. PMS events represent the segregation of two alleles from one meiotic product postmeiotically. If the mutant allele is an auxotrophic mutation, PMS events are signaled by sectored colonies formed when spore colonies grown on rich medium are replica plated to omission medium. In $S$. cerevisiae, the most common types of PMS tetrads are those with $2 A: 1 a: 1$ sectored $A / a$ colony or $1 A: 2 a: 1$ sectored $A / a$ colony. Since comparable patterns of PMS were first detected in eight-spored fungi (in which the meiotic divisions are followed by a single mitotic division), these patterns are described as $5 A: 3 a$ or $3 \mathrm{~A}: 5 a$ patterns, respectively. To use a consistent nomenclature, we will describe gene conversion events as $6: 2$ or $2: 6$ and normal Mendelian segregation as 4:4.

In $S$. cerevisiae, tetrads with a single PMS event $(5: 3$ or $3: 5)$ are usually much more common than tetrads with two PMS events (aberrant $4: 4$, aberrant $6: 2$, or aberrant $2: 6$ ) (7). Single PMS tetrads presumably reflect the nonreciprocal transfer of one strand of DNA between the two alleles. For example, if one strand of DNA is donated from the $A$ allele to the $a$ allele, a heteroduplex will be formed. If the resulting mismatch is not repaired, one would expect to get a $5 A: 3 a$ pattern of segregation. Similarly, a $3 A: 5 a$ segregation pattern would result from an unrepaired mismatch in a heteroduplex formed as the result of transfer of a single strand of DNA from $a$ to $A$. The relatively small number of tetrads with two PMS events in $S$. cerevisiae suggests that heteroduplex formation is usually asymmetric $(16,25)$ rather than symmetric (11).

Although all PMS events are thought to reflect the formation of heteroduplexes, two different hypotheses have been suggested to explain the origin of gene conversion events. In one class of models, the conversion events are the result of repair of mismatches in heteroduplex DNA $(10,11,16,25)$.

\footnotetext{
* Corresponding author
}

An alternative model is that gene conversions reflect repair of a double-stranded gap with sequences from a homologous chromatid (30). There are several lines of evidence suggesting that most meiotic conversion is the result of mismatch repair. First, the frequency of PMS per aberrant segregation depends to a large extent on the type of mismatch that the heterozygous alleles form. For example, two of the highPMS alleles in yeast are G-to-C mutations; other base substitution alleles show less PMS $(7,31)$. Second, mutant alleles have been identified that elevate PMS and depress gene conversion, as though these events represent alternative processing of the same intermediate. For example, a mutant allele resulting from insertion of a 26-bp palindromic oligonucleotide into the $H I S 4$ gene has a very high frequency of PMS and a low frequency of conversion relative to a mutant allele resulting from an insertion of 4 bp into HIS4 at the same position (20). Third, there are DNA repair mutants of $S$. cerevisiae that (for some alleles) increase the frequency of PMS while decreasing the frequency of gene convcrsion $(1,2,13,14,32)$.

Conventional tetrad analysis has limitations for assessing the frequency of repair of a particular mismatch. Because of the double-stranded nature of DNA, a diploid heterozygous for a mutant allele can form two different types of mismatches. For example, a wild-type gene has the initiating codon 5'-ATG in the nontranscribed strand and the complementary sequence $5^{\prime}$-CAT on the other strand. Heteroduplexes formed between this wild-type allele and a mutant allele with the sequence 5'-ATC (complementary strand 5'-GAT) would be of two types (Fig. 1). If the nontranscribed strand is donated from the wild type to the mutant, or the transcribed strand is donated from the mutant to the wild type, a $\mathrm{G} / \mathrm{G}$ mismatch would result. If the transcribed strand is donated from the wild type to the mutant, or the nontranscribed is donated from mutant to wild type, a $\mathrm{C} / \mathrm{C}$ mismatch would result. Conventional tetrad analysis, therefore, can only indicate the frequency of PMS for pairs of mismatches. For base substitution mutations, there are four possible mismatch pairs: C/C-G/G, A/A-T/T, G/T-A/C, and G/A-T/C. The reported rates of PMS per aberrant segregation in $S$. cerevisiae are 33 to $48 \%$ for $\mathrm{C} / \mathrm{C}-\mathrm{G} / \mathrm{G}$ and 3 to $8 \%$ for the other three pairs $(7,13,31)$. From studies of the 
a. $5^{+}: 3^{-}$

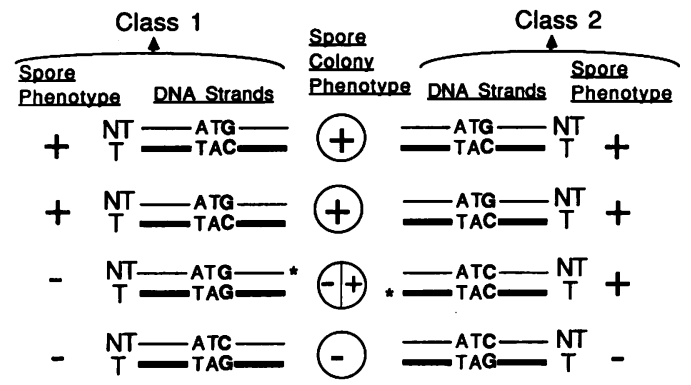

b. $3^{+}: 5^{-}$

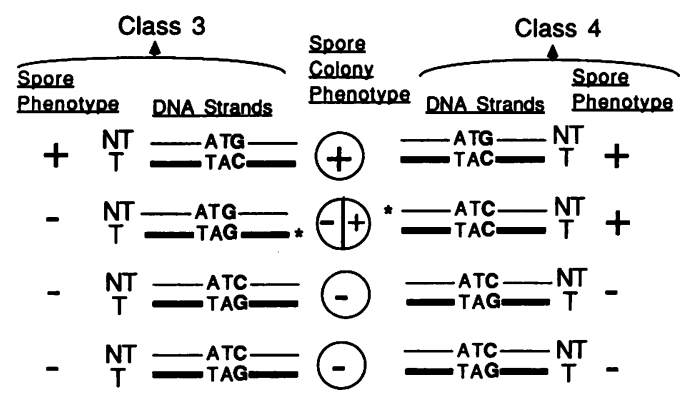

FIG. 1. Expected base mismatches in heteroduplexes formed between wild-type (ATG) and mutant (ATC) his 4 alleles. The configurations of DNA molecules expected from both $5^{+}: 3^{-}$(a) and $3^{+}: 5^{-}$(b) tetrads are shown. A mutation in the first ATG sequence (encoding the AUG initiating codon) in HIS4 to ATC leads to a His ${ }^{-}$ phenotype (5). This mutation leads to either a $\mathrm{C} / \mathrm{C}$ or $\mathrm{G} / \mathrm{G}$ mismatch, depending on whether the donor strand is wild type or mutant and transcribed or nontranscribed. For example, if the wild-type gene donates a nontranscribed strand, the resulting mismatch is $G / G$ (class 1). The mismatches for the other classes are also shown. The donated strands are indicated by asterisks.

frequency of heteroallelic recombination at the $C Y C 1$ locus, Moore et al. (18) also concluded that either the $\mathrm{C} / \mathrm{C}$ or the G/G mismatch was corrected inefficiently relative to other point mismatches.

The mitotic repair of individual mismatches has been studied by using heteroduplexes constructed in vitro. Such heteroduplexes were transformed into vegetative haploid yeast cells. The transformed cells were allowed to form colonies that were subsequently examined for segregation of both alleles (indicating failure of mismatch repair). Kramer et al. (13) found that the $\mathrm{C} / \mathrm{C}$ mismatch evaded repair to a greater extent than the other base substitution mismatches; insertions of single base pairs were the most efficiently repaired. Using similar methods, Bishop et al. (1) concluded that $\mathrm{C} / \mathrm{C}, \mathrm{A} / \mathrm{A}$, and $\mathrm{T} / \mathrm{T}$ mismatches were repaired less efficiently than $A / C, G / T, A / G$, and $G / G$ mismatches or single-base-pair insertions; the $\mathrm{C} / \mathrm{T}$ mismatch was repaired with intermediate efficiency. Similar studies in procaryotes indicate that $\mathrm{C} / \mathrm{C}$ is poorly repaired relative to other mismatches $(4,17,26)$.

Although these heteroduplex transformation studies are consistent with the possibility that the high frequency of PMS observed with mutations resulting in a G/G-C/C mismatch is a consequence of poor repair of the $\mathrm{C} / \mathrm{C}$ mismatch, the studies are not definitive for two reasons. First, these previous studies concern mitotic rather than meiotic mismatch repair. Second, it has been proposed that the way in
TABLE 1. Yeast strains and plasmids used to construct the strains

\begin{tabular}{lcll}
\hline $\begin{array}{c}\text { Diploid } \\
\text { strain }\end{array}$ & $\begin{array}{c}\text { Haploids mated } \\
\text { to form diploid }\end{array}$ & $\begin{array}{c}\text { Relevant genotype } \\
\text { of diploid }\end{array}$ & $\begin{array}{c}\text { Plasmid used to } \\
\text { insert his4 mutation }\end{array}$ \\
\hline PD82 & PD72 $\times$ AS4 & his4-CTG/HIS4 & p44 \\
PD83 & PD73 $\times$ AS4 & his4-AAG/HIS4 & p659 \\
PD84 & PD74 $\times$ AS4 & his4-ATC/HIS4 & p42 \\
JS102 & JS2 $\times$ AS13 & HIS4/his4-ATC & p42 \\
PD85 & PD75 $\times$ AS4 & his4-ACG/HIS4 & p65 \\
PD86 & PD76 $\times$ AS4 & his4-GTG/HIS4 & p76 \\
PD87 & PD77 $\times$ AS4 & his4-AGG/HIS4 & p661 \\
PD88 & PD78 $\times$ AS4 & his4-ATT/HIS4 & p74 \\
PD89 & PD79 $\times$ AS4 & his4-TTG/HIS4 & p169 \\
JS101 & JS1 $\times$ AS4 & his4-ATA/HIS4 & p49 \\
PD9 & PD5 $\times$ AS4 & his4-519/HIS4 & phis4-519 \\
PD11 & PD6 $\times$ AS4 & his4-17/HIS4 & phis4-17 \\
\hline
\end{tabular}

which a mismatch is repaired is dependent on which strand is transferred in formation of the meiotic heteroduplex (9).

Lichten et al. (15) used a method of gel electrophoresis that allows detection of individual mismatches in heteroduplexes in DNA isolated from sporulated yeast cells. They conclude from this analysis that a $\mathrm{C} / \mathrm{C}$ mismatch in the $A R G 4$ gene was repaired at least fivefold less efficiently than a $G / G$ mismatch. We recently developed a genetic method of identifying which specific mismatch of a pair is left unrepaired in individual PMS events (19). As described below, we used this technique, in addition to conventional tetrad analysis, to analyze the repair of all eight single-base-pair mismatches. We concluded that $\mathrm{C} / \mathrm{C}$ mismatches are repaired by the meiotic repair system about threefold less efficiently than all other mismatches. In addition, we found (in confirmation of previous studies by Lichten et al. [15] and Nag and Petes [19]) that most unrepaired mismatches are in heteroduplexes in which the nontranscribed strand was the donor.

\section{MATERIALS AND METHODS}

Plasmids and yeast strains. The plasmids containing mutations in the translation initiation site of HIS4 were gifts from M. Cigan and T. Donahue. Their construction was described previously (5). In brief, p42, p44, p49, p65, p74, p76, and p169 contain mutations in the ATG initiating codon (Table 1) located within a 760-bp Sau3AI fragment of the upstream region of HIS4; this fragment is inserted into the BamHI site of B142 (YIp5 without a PvuII site; 5). Plasmids p659 and p661 have the mutant ATG substitutions within a 1.5-kb SalI fragment containing the $5^{\prime}$ end of the HIS4 gene; this fragment is inserted into the SalI site of B142.

Plasmids phis4-519 (B137) and phis4-17 (B135) were gifts from B. Ruskin and G. Fink. These plasmids contain mutant his4 genes on an EcoRI-BamHI fragment inserted into EcoRI and BamHI sites of YIp5. The his4-519 mutation is a insertion of a single $G$ near position $+493(+1$ is the translation start site) (6). The his4-17 mutation is a T-to-C base substitution at position +688 (6).

The yeast strains used in this study were derivatives of AS4 (MATa trpl arg4 tyr7 ade6 ura3 MAL2) and AS13 (MATa leu2 ade6 ura3). These strains were constructed by A. Stapleton from the haploid strains LS18 and LS25-70d (29) by multiple transformations. In addition to auxotrophic markers, these strains contain multiple restriction site changes between $L E U 2$ and $C E N 3$. The diploid strains used in our study were isogenic (except for changes introduced by 
transformation) with a diploid produced by crossing AS4 and AS13. Table 1 shows the plasmids and strains used. For most constructions, the wild-type HIS4 gene in the haploid AS13 was replaced by a mutant gene in the two-step transplacement procedure $(27,33)$; for strain JS2, the wild-type HIS4 gene in AS4 was replaced by a mutant his4 gene. For the first step of the transplacement, various plasmids with the URA3 gene and a mutant his 4 gene were transformed into the host strain, and $\mathrm{Ura}^{+}$cells were selected. For this step, all plasmids except phis4-519 and phis4-17 were treated with PvuII to target the integration (22); phis4-519 and phis4-17 were treated with SpeI. Subsequently, $\mathrm{Ura}^{-}$derivatives of the transformed strains were selected by using 5-fluoroorotate (3) and screened for those derivatives that were also $\mathrm{His}^{-}$. The resulting haploid strains were mated to AS4 or AS13 to generate the diploids shown in Table 1.

Media and genetic techniques. Most of the genetic procedures used were standard (28) except for the altered procedure of tetrad analysis. Cells were grown vegetatively in YPD medium (1\% yeast extract, 2\% Bacto-Peptone [Difco], $2 \%$ dextrose) overnight and were then transferred to plates containing sporulation medium ( $1 \%$ potassium acetate, $0.1 \%$ yeast extract, $0.05 \%$ dextrose, $2 \%$ agar) supplemented with adenine $(6 \mu \mathrm{g} / \mathrm{ml})$. These plates were incubated at $18^{\circ} \mathrm{C}$ until tetrads had formed ( 3 to 5 days). Tetrads were treated with Glusulase and dissected. For the conventional tetrad analysis, the spores were dissected on YPD plates and grown for 2 to 3 days at $32^{\circ} \mathrm{C}$, and the resulting spore colonies were replica plated to various types of omissic. n media. After 1 day, colonies on the plates lacking histidil.e were examined with the microscope (25-fold magnification) to detect small sectors; by this procedure, sectors smaller than $5 \%$ of the colony could be easily detected.

For the altered tetrad dissection protocol (19), the sporulated asci were dissected onto SD-his plates (solid medium containing $0.17 \%$ Difco yeast nitrogen base [without ammonium sulfate or amino acids], $0.5 \%$ ammonium sulfate, $2 \%$ dextrose, $2 \%$ agar, and all required amino acids and bases [concentration of $0.1 \mathrm{mg} / \mathrm{ml}$ ] except histidine). The dissected spores were incubated at $32^{\circ} \mathrm{C}$ for about $9 \mathrm{~h}$ and were then examined microscopically. On SD-his plates, spores containing a mutant his 4 gene or a gene with mutant information on the transcribed strand and wild-type information on the nontranscribed strand fail to divide. Spores with a wild-type HIS4 gene or a gene with wild-type information in the transcribed strand and mutant information in the nontranscribed strand begin dividing on SD-his medium. Thus, in most of the tetrads, two of the spores failed to divide or bud and the other two spores had divided three times (6); all spores that had budded or divided one or more times were scored as $\mathrm{His}^{+}$. After scoring all spores, we transferred the agar slab containing the spores on top of a slab of solid YPD medium that was overlaid with $0.4 \mathrm{ml}$ of a $0.5 \%$ histidine solution. The histidine diffused from one slab into the other, allowing nonselective growth of both $\mathrm{His}^{+}$and $\mathrm{His}^{-}$cells. The plates were incubated at $32^{\circ} \mathrm{C}$ for 3 to 4 days and then replica plated to SD-his medium (as well as to other omission media). After 1 day, all colonies on the SD-his plate were examined with the microscope to detect small sectors. The pattern of sectoring was then correlated with the phenotype of the spore. As described below, this protocol allows one to determine which mismatched bases in the HIS4 gene are responsible for the sectored colony.

Statistical methods. The statistical comparisons were usually done by using $2 \times 2$ contingency chi-square analysis. A chi-square value greater than 3.85 was considered significant at the $\boldsymbol{P}=\mathbf{0 . 0 5}$ level. In comparisons in which one or more classes had fewer than five events, we determined $\boldsymbol{P}$ values by the Fisher exact test, using the StatXact program (Cytel Software Corp.).

\section{RESULTS}

Experimental system. We examined the meiotic segregation of heterozygous mutations in the initiating AUG codon of the HIS4 gene. This particular system was chosen for several reasons. First, in our strain background, mutations at the HIS4 locus have an extraordinarily high level of aberrant segregation (20); this high rate of aberrant segregation occurs only at the HIS4 locus (20). Second, most of the aberrant segregations at this locus have a heteroduplex intermediate (20). Third, the HIS4 gene and many mutant derivatives of this gene have been sequenced (6). In particular, Donahue and Cigan (5) have constructed all nine possible single-base-pair mutations in the DNA sequence encoding the AUG initiating codon ( +1 ATG). The frequency of aberrant segregations at the HIS4 locus is higher for substitutions located near the $5^{\prime}$ end of the gene than for those near the $3^{\prime}$ end. Such polarity gradients have also been observed for several other yeast genes (such as $A R G 4 ; 7$ ). Thus, mutational alterations in the +1 ATG sequence would be expected to have a particularly high frequency of aberrant segregation. In addition, since all mutations in the +1 ATG sequence are within three base pairs, any effects related to the position of the mutation within the gene would be expected to be minimal.

Rates of PMS in strains heterozygous for various mutational alterations. Strains heterozygous for various mutational alterations at the HIS4 locus (otherwise isogenic) were sporulated, and tetrads were dissected onto plates containing rich growth medium. After spore colonies were formed, the plates were replica plated to plates containing various types of omission media. After overnight growth, the segregation of all heterozygous markers was examined. The colonies that were growing on plates lacking histidine were examined microscopically to ensure that all sectors could be detected.

The patterns of aberrant segregation at the HIS4 locus in these strains are shown in Table 2 , and the frequencies of PMS for various mutant alleles are summarized in Table 3. All strains except PD9 and PD11 contain mutations in the ATG sequence representing the initiating AUG codon of HIS4. PD9 has the his4-519 mutation, an insertion of a single base pair ( $\mathrm{G}$ in the nontranscribed strand) between +493 and +495 (6), whereas PD11 contains the his4-17 mutation, a T-to-C change in position +688 . The aberrant segregation rate of HIS4 mutations in the ATG sequence was extremely high, about $50 \%$ of unselected tetrads. Except for the HIS4 gene, the reported rates of abberant segregation for alleles in $S$. cerevisiae vary between 0.6 and $18 \%$, with a median frequency of about $4 \%$ (7). Mutations at the $5^{\prime}$ end of the HIS4 gene had a higher frequency of aberrant segregation than those (his4-17 and his4-519) toward the 3' end, confirming the polarity gradient previously observed (31a). Since mutations at the same position in the gene have similar rates of aberrant segregation and since most gene conversion events at HIS4 have a heteroduplex intermediate (20), we assume that the frequency of heteroduplex formation is the same for mismatches at the same position in HIS4.

Since the frequency of aberrant segregation was very high for these mutations, it is not surprising that some tetrads had the segregation patterns expected as the result of two recombination events. These tetrads may be interpreted as 
TABLE 2. Numbers and types of aberrant segregations of different his4 mutant alleles

\begin{tabular}{|c|c|c|c|c|c|c|c|c|c|c|c|c|c|}
\hline \multirow{3}{*}{$\begin{array}{l}\text { Diploid } \\
\text { strain }\end{array}$} & \multirow{3}{*}{$\begin{array}{l}\text { Mutant } \\
\text { allele }\end{array}$} & \multicolumn{10}{|c|}{ Segregation } & \multirow{3}{*}{$\begin{array}{l}\text { Total } \\
\text { tetrads }\end{array}$} & \multirow{3}{*}{$\begin{array}{l}\text { Aberrant } \\
\text { segregation } \\
\text { tetrads } \\
\text { (\% of total) }\end{array}$} \\
\hline & & \multicolumn{9}{|c|}{ Aberrant } & \multirow{2}{*}{ Others $^{a}$} & & \\
\hline & & $5^{+}: 3^{-}$ & $3^{+}: 5^{-}$ & $7^{+}: 1^{-}$ & $1^{+}: 7^{-}$ & $4^{+}: 4^{-}$ & $6^{+}: 2^{-}$ & $2^{+}: 6^{-}$ & $8^{+}: 0^{-}$ & $0^{+}: 8^{-}$ & & & \\
\hline $\mathrm{PD}^{2} 2^{b}$ & his4-CTG & 18 & 15 & 3 & 7 & 8 & 99 & 114 & 20 & 29 & 0 & 640 & 49 \\
\hline PD83 & his4-AAG & 11 & 10 & 1 & 2 & 0 & 54 & 58 & 13 & 23 & 1 & 315 & 55 \\
\hline PD84 ${ }^{b}$ & his4-ATC & 56 & 57 & 6 & 4 & 3 & 113 & 33 & 21 & 1 & 8 & 677 & 45 \\
\hline JS102 & his4-ATC & 22 & 21 & 3 & 3 & 10 & 46 & 18 & 9 & 2 & 0 & 256 & 52 \\
\hline $\mathrm{PD}^{b} 5^{b}$ & his4-ACG & 12 & 25 & 0 & 6 & 2 & 132 & 156 & 14 & 23 & 1 & 784 & 47 \\
\hline PD86 & his4-GTG & 8 & 9 & 2 & 2 & 0 & 63 & 88 & 4 & 13 & 0 & 405 & 47 \\
\hline PD87 & his4-AGG & 10 & 8 & 0 & 1 & 1 & 55 & 79 & 11 & 13 & 0 & 339 & 53 \\
\hline PD88 & his4-ATT & 8 & 9 & 2 & 2 & 0 & 31 & 52 & 4 & 14 & 2 & 295 & 42 \\
\hline PD89 $^{b}$ & his4-TTG & 16 & 18 & 6 & 5 & 4 & 92 & 109 & 15 & 13 & 1 & 598 & 47 \\
\hline JS101 & his4-ATA & 8 & 6 & 3 & 1 & 0 & 42 & 58 & 10 & 9 & 0 & 311 & 44 \\
\hline PD9 & his4-519 & 0 & 0 & 0 & 0 & 0 & 29 & 60 & 1 & 0 & 0 & 311 & 29 \\
\hline PD11 & his4-17 & 6 & 12 & 0 & 0 & 1 & 23 & 44 & 3 & 1 & 0 & 272 & 33 \\
\hline
\end{tabular}

${ }^{a}$ Includes aberrant 6:2 and aberrant $2: 6$, as well as tetrads with more than two PMS events.

$b$ Includes data from both conventional and altered methods of tetrad analysis.

follows: 8:0 and 0:8 (two conversion events), 7:1 and 1:7 (one conversion and one PMS event), aberrant 4:4 (two PMS events with one wild-type donor and one mutant donor), aberrant 6:2 (two PMS events with two wild-type donors), and aberrant 2:6 (two PMS events with two mutant donors). Most of these classes were observed at approximately the frequency expected for two independent events. In particular, the frequency of aberrant 4:4 tetrads was lower than the frequency of 5:3 and 3:5 tetrads for most of the strains, indicating that most of the heteroduplexes were formed asymmetrically $(7,20)$.

The his4-ATC mutation in PD84 and JS102 showed higher PMS than any of the other mutant alleles (Table 3); in these strains $44 \%$ of all aberrant segregations had one or more PMS events. This frequency is significantly higher than that observed for any of the other strains with base substitution mutant alleles in the HIS4 translation initiation site (contingency chi-square values of 21 or more). In these strains, the frequencies of PMS were in the range of 11 to $19 \%$ of the aberrant segregations and 5 to $8 \%$ of the total tetrads. With the exception of his4-ATC, none of the HIS4 ATG mutations showed significant differences between their rates of PMS per tetrad or PMS per aberrant segregation. The his4-519

TABLE 3. Rates of PMS for different mismatch pairs at HIS4

\begin{tabular}{|c|c|c|c|c|}
\hline Mismatch pair & Strain & $\begin{array}{l}\text { PMS/aberrant } \\
\text { segregation (\%) }\end{array}$ & $\begin{array}{l}\text { PMS/total } \\
\text { tetrads (\%) }\end{array}$ & $\begin{array}{l}\text { Position of } \\
\text { mutation }^{a}\end{array}$ \\
\hline \multirow[t]{2}{*}{$\mathrm{T} / \mathrm{T}-\mathrm{A} / \mathrm{A}$} & PD83 & 15 & 8 & +2 \\
\hline & PD89 $^{b}$ & 18 & 8 & +1 \\
\hline \multirow[t]{4}{*}{ T/G-C/A } & PD86 & 11 & 5 & +1 \\
\hline & JS101 & 13 & 6 & +3 \\
\hline & PD85 $^{b}$ & 12 & 6 & +2 \\
\hline & PD11 & 21 & 7 & +688 \\
\hline \multirow[t]{3}{*}{ T/C-G/A } & PD87 & 11 & 6 & +2 \\
\hline & PD88 & 19 & 8 & +3 \\
\hline & $\mathrm{PD82}^{b}$ & 16 & 8 & +1 \\
\hline \multirow[t]{2}{*}{ G/G-C/C } & PD84 ${ }^{b}$ & 44 & 20 & +3 \\
\hline & JS102 & 44 & 23 & +3 \\
\hline $\begin{array}{l}\text { Insertion of } \\
\text { G-insertion } \\
\text { of C }\end{array}$ & PD9 & 0 & 0 & +493 \\
\hline
\end{tabular}

$a+1$ indicates the base pair encoding the first base of the initiating AUG codon.

${ }^{b}$ Includes tetrads from conventional and altered tetrad analysis. allele, which is an insertion of a single $G$ about $493 \mathrm{bp}$ downstream of the translation initiation site, showed no PMS. This frequency was significantly less than observed for all other base substitution alleles in this study (Fisher exact tests in comparisons with other strains gave $\boldsymbol{P}$ values of 0.0002 or less).

The higher level of PMS for the his4-ATC mutation is probably due to the nature of the mismatches formed in meiotic heteroduplexes. Since two other mutations at the same position (+3) in the HIS4 gene do not have a high level of PMS, this observation does not reflect a context-specific feature of the repair system. PD84 and JS102 are the only strains analyzed that would form $\mathrm{G} / \mathrm{G}$ or $\mathrm{C} / \mathrm{C}$ mismatches in heteroduplexes between the wild-type and mutant HIS4 genes. Thus, our results confirm previous studies $(7,13,21$, 31 ) indicating that this mismatch pair results in high PMS. To determine the rates of repair of individual mismatches, we used an alternative tetrad dissection procedure.

Rate of meiotic repair of individual mismatches. The frequency of repair of the mismatch is assumed to be inversely related to the frequency of PMS. If the mutation is a G-to-C change in the ATG sequence at the beginning of the HIS4 gene, transfer of the nontranscribed strand in a $5^{+}: 3^{-}$tetrad would reflect a $G / G$ mismatch, whereas transfer of the transcribed strand would reflect a C-C mismatch (Fig. 1). For $3^{+}: 5^{-}$tetrads, transfer of the nontranscribed strand would result in a $\mathrm{C} / \mathrm{C}$ mismatch and transfer of the transcribed strand would result in a G/G mismatch.

To determine whether a transcribed or nontranscribed strand was donated and which mismatch was left unrepaired, we dissected the heterozygous strains onto medium lacking histidine (19). After about $9 \mathrm{~h}$, the spores were observed microscopically and scored as $\mathrm{His}^{+}$(the spore had undergone cell division) or $\mathrm{His}^{-}$(no cell division). Spores scored as $\mathrm{His}^{+}$presumably have wild-type HIS4 information in the transcribed strand, and spores scored as $\mathrm{His}^{-}$presumably have mutant information in the transcribed strand. The agar slab containing the spores was transferred to a plate containing agar with excess histidine. The diffusion of histidine into the agar originally lacking histidine allows both $\mathrm{His}^{+}$and $\mathrm{His}^{-}$cells to form a colony. The resulting colonies were then replica plated to medium lacking histidine. If a PMS event for the 5:3 class is observed and the sectored colony was derived from a $\mathrm{His}^{-}$spore (class 1 ), we can conclude that the 
TABLE 4. Segregation patterns for tetrads with PMS events at the HIS4 locus

\begin{tabular}{|c|c|c|c|c|c|c|}
\hline \multirow{2}{*}{$\begin{array}{l}\text { Segregation } \\
\text { pattern }^{a}\end{array}$} & \multirow{2}{*}{$\begin{array}{l}\text { His phenotype of } \\
\text { PMS spore(s) }\end{array}$} & \multirow{2}{*}{$\begin{array}{l}\text { Class of } \\
\text { sectored colony }\end{array}$} & \multicolumn{4}{|c|}{ No. of tetrads ${ }^{b}$} \\
\hline & & & PD82 & PD84 & PD85 & PD89 \\
\hline $\begin{array}{l}5^{+}: 3^{-} \\
0000\end{array}$ &,-+ & 1,2 & 9,2 & 9,18 & 6,0 & 5,3 \\
\hline $\begin{array}{l}3^{+}: 5^{-} \\
0000\end{array}$ &,-+ & 3,4 & 4,8 & 5,29 & 1,10 & 1,7 \\
\hline $7^{+}: 1^{-}$ &,-+ & 1,2 & 1,0 & 3,0 & 0,0 & 1,1 \\
\hline $\begin{array}{l}1^{+}: 7^{-} \\
0000\end{array}$ &,-+ & 3,4 & 1,1 & 0,3 & 0,3 & 0,3 \\
\hline $\begin{array}{l}\text { Aberrant } 4^{+}: 4^{-} \\
\text {- }\end{array}$ & $-I-,+1-,+1+$ & & $1,1,0$ & $0,3,0$ & $0,1,0$ & $1,0,1$ \\
\hline $\begin{array}{l}\text { Aberrant } 6^{+}: 2^{-} \\
000\end{array}$ & $-1-,+1-,+1+$ & $1 / 1,2 / 1,2 / 2$ & $0,0,0$ & $0,0,1$ & $0,0,0$ & $0,0,0$ \\
\hline $\begin{array}{l}\text { Aberrant } 2^{+}: 6^{-} \\
0000\end{array}$ & $-1-,+1-,+1+$ & $3 / 3,4 / 3,4 / 4$ & $0,0,0$ & $0,0,0$ & $0,0,0$ & $0,0,0$ \\
\hline Other ${ }^{c}$ & & & 0 & $1^{c}$ & 0 & 0 \\
\hline
\end{tabular}

${ }^{a}$ Below the numerical representation of the segregation pattern is a schematic representation of spore colonies. The dark color represents wild-type (His $\left.{ }^{+}\right)$ cells.

${ }^{b}$ Obtained from dissection by the altered dissection protocol and having one or two PMS events per tetrad.

${ }^{c}$ Deviant 3:5 tetrad ( 3 sectored spore colonies: 1 His $^{-}$spore colony). Two of the sectors were derived from His ${ }^{+}$spores, and one was derived from a His ${ }^{-}$ spore.

sector resulted from transfer of the nontranscribed strand from wild type to mutant and an unrepaired $\mathrm{G} / \mathrm{G}$ mismatch (Fig. 1). Similar arguments can be used to explain the other three classes shown in Fig. 1. It should be pointed out that all sectors derived from $\mathrm{His}^{-}$spores represent $\mathrm{G} / \mathrm{G}$ mismatches and all derived from $\mathrm{His}^{+}$spores represent $\mathrm{C} / \mathrm{C}$ mismatches.

Some of the classes of tetrads with more than one PMS event or a conversion event and a PMS event can also be classified as representing class $1,2,3$, or 4 events. For example, an aberrant 6:2 tetrad (two $\mathrm{His}^{+}$spore colonies: 2 sectored $\mathrm{His}^{+} / \mathrm{His}^{-}$spore colonies) in which the sectored colonies are derived from $\mathrm{His}^{+}$spores can be classified as representing two class 2 events. Tetrads of the aberrant 4:4 class cannot be unambiguously classified since it is unclear whether the individual sectored colonies were the result of transfer of a mutant strand to a wild-type gene or vice versa. Thus, sectors derived from such tetrads cannot be used to determine whether a transcribed or nontranscribed strand was donated, although they can be used to determine which mismatch was left unrepaired.

The analyses for strains PD84 (C/C-G/G mismatches), PD82 (G/A-T/C mismatches), PD85 (T/G-C/A mismatches), and PD89 (A/A-T/T mismatches) are summarized in Table 4. The expected mismatches for each heteroduplex of these strains are shown in Table 5, and the unrepaired individual mismatches are summarized in Table 6. Assuming the same frequency of heteroduplex formation for all mismatches, we conclude that the $\mathrm{C} / \mathrm{C}$ mismatch was repaired less efficiently than any of the other mismatches (including G/G). The chi-square values were 15.7 for the $\mathrm{C} / \mathrm{C}-\mathrm{G} / \mathrm{G}$ comparison and greater than 16.8 for the comparisons of $C / C$ with any of the other mismatches. All other mismatches (A/A, T/T, T/G, $\mathrm{C} / \mathrm{A}, \mathrm{G} / \mathrm{A}, \mathrm{T} / \mathrm{C}$, and $\mathrm{G} / \mathrm{G}$ ) were repaired with approximately the same efficiency. The only exception to this generalization is that the level of unrepaired mismatches for $G / G$ was significantly greater (chi-square value of 4.8) than for $T / G$. Table 7 is a comparison of the classes of aberrant segregation in strains dissected by conventional procedures and by a modified protocol. It is clear that these two procedures have similar distributions of the classes of aberrant segregation.
Preferential donation of the nontranscribed strand in heteroduplexes with unrepaired mismatches. The number of PMS tetrads in the four classes shown in Fig. 1 is a function of two variables: (i) the relative frequency of transfer of the transcribed and nontranscribed strands and (ii) the efficiency of repair of the individual mismatches. The contributions of these two factors to the frequency of the four classes can be calculated separately (19). In Table 8, we tabulate the number of sectors resulting from transfer of the transcribed and nontranscribed strands. For all four strains, there were significantly (all chi-square values greater than 4.6) more sectors resulting from transfer of the nontranscribed strand than from transfer of the transcribed strand. Although the transcribed strand/nontranscribed strand ratio varied somewhat for different strains, only the difference in the ratios for strains PD84 and PD85 was statistically significant $(P=$

TABLE 5. Expected mismatches formed as the result of heteroduplex formation between wild-type and mutant his 4 alleles

\begin{tabular}{|c|c|c|c|c|}
\hline Strain & $\begin{array}{l}\text { Mutant } \\
\text { allele }^{a}\end{array}$ & $\begin{array}{c}\text { Expected } \\
\text { heteroduplexes } \\
\text { between wild-type } \\
\text { and mutant alleles }\end{array}$ & $\begin{array}{l}\text { His phenotype } \\
\text { of spore }\end{array}$ & Mismatch \\
\hline \multirow[t]{2}{*}{ PD89 } & his4-TTG & $\begin{array}{l}5^{\prime} \text {-ATG-3' } \\
3^{\prime} \text {-AAC-5' }\end{array}$ & - & $\mathbf{A} / \mathbf{A}$ \\
\hline & & $\begin{array}{l}\text { 5'-TTG-3' } \\
\text { 3'-TAC-5' }\end{array}$ & + & $T / T$ \\
\hline \multirow[t]{2}{*}{ PD85 } & his4-ACG & $\begin{array}{l}5^{\prime}-\text { ATG-3' } \\
3^{\prime}-\text { TGC-5' }\end{array}$ & - & $T / G$ \\
\hline & & $\begin{array}{l}5^{\prime}-\mathrm{ACG}-3^{\prime} \\
3^{\prime}-\mathrm{TAC}-5^{\prime}\end{array}$ & + & C/A \\
\hline \multirow[t]{2}{*}{ PD82 } & his4-CTG & $\begin{array}{l}\text { 5'-ATG-3' } \\
3^{\prime}-\text { GAC-5' }\end{array}$ & - & G/A \\
\hline & & $\begin{array}{l}5^{\prime}-\mathrm{CTG}-3^{\prime} \\
3^{\prime}-\mathrm{TAC}-5^{\prime}\end{array}$ & + & $T / C$ \\
\hline PD84 & his4-ATC & $\begin{array}{l}\text { 5'-ATG-3' } \\
\text { 3'-TAG-5' }\end{array}$ & - & $\mathrm{G} / \mathrm{G}$ \\
\hline & & $\begin{array}{l}\text { 5'-ATC-3' } \\
3^{\prime}-\text { TAC-5' }\end{array}$ & + & $\mathrm{C} / \mathrm{C}$ \\
\hline
\end{tabular}

\footnotetext{
${ }^{a}$ Wild type is his4-ATG.
} 
TABLE 6. Number of sectored colonies resulting from specific unrepaired mismatches

\begin{tabular}{cccccc}
\hline Strain & $\begin{array}{c}\text { Total } \\
\text { no. of } \\
\text { tetrads }\end{array}$ & $\begin{array}{c}\text { Total } \\
\text { no. of } \\
\text { sectors }\end{array}$ & $\begin{array}{c}\text { Expected } \\
\text { mismatch }\end{array}$ & $\begin{array}{c}\text { No. of sectors } \\
\text { resulting from } \\
\text { mismatch }\end{array}$ & $\begin{array}{c}\text { Sectors due to } \\
\text { mismatch/total } \\
\text { tetrads (\%) }\end{array}$ \\
\hline PD89 & 315 & 25 & A/A & 9 & 3 \\
PD85 & 385 & 23 & T/G & 16 & 5 \\
& & & C/A & 14 & 2 \\
PD82 & 327 & 30 & G/A & 18 & 4 \\
& & & T/C & 12 & 6 \\
PD84 & 367 & 78 & G/G & 21 & 4 \\
& & & C/C & 57 & 16 \\
\hline
\end{tabular}

0.011 by the Fisher exact test). The conclusion that the nontranscribed strand is transferred more frequently than the transcribed strand in heteroduplexes with unrepaired mismatches is consistent with previous observations at the HIS4 (19) and ARG4 (15) loci.

\section{DISCUSSION}

When a heteroduplex containing a mismatch is formed during meiotic recombination, there are three possible consequences: (i) the mismatch may remain unrepaired, leading to a PMS event; (ii) the mismatch may be repaired in the direction of the invading strand, leading to a gene conversion event; or (iii) the mismatch may be repaired in the direction of the invaded strand, leading to a restoration event. Our study, therefore, concerns two issues, the efficiency of repair and the direction of repair. These issues are discussed separately below.

$\mathrm{C} / \mathrm{C}$ mismatches are less efficiently repaired in meiotic heteroduplexes than any other single-base-pair mismatch. Our analysis indicates that $\mathrm{C} / \mathrm{C}$ mismatches are less efficiently repaired in meiosis than any of the other seven base pair mismatches. All other mismatches are repaired with approximately the same efficiency. A single-base-pair insertion was repaired more efficiently than any of the mismatches resulting from base substitutions. Our results are qualitatively in agreement with those of other researchers, although there are several interesting quantitative differences. The arg4-16 mutation (which would result in a $\mathrm{C} / \mathrm{C}$ or $\mathrm{G} / \mathrm{G}$ mismatch in a heteroduplex with a wild-type strand) had a PMS frequency of $32.5 \%$ of the aberrant tetrads, whereas mutations resulting in $\mathrm{A} / \mathrm{A}-\mathrm{T} / \mathrm{T}$ or $\mathrm{A} / \mathrm{C}-\mathrm{G} / \mathrm{T}$ mismatches had PMS frequencies of 5 and $4 \%$, respectively $(7,31,32)$. Williamson et al. (32) found no PMS for strains heterozygous for the his4-519
TABLE 8. Number of sectors formed by the transfer of transcribed and nontranscribed strands ${ }^{a}$

\begin{tabular}{lccc}
\hline Strain & $\begin{array}{c}\text { Total no. of } \\
\text { tetrads }\end{array}$ & \multicolumn{2}{c}{ Sectors derived from transfer of: } \\
\cline { 3 - 4 } & $\begin{array}{c}\text { Nontranscribed } \\
\text { strand }\end{array}$ & $\begin{array}{c}\text { Transcribed } \\
\text { strand }\end{array}$ \\
\hline PD82 & 327 & 19 & 7 \\
PD84 & 367 & 46 & 23 \\
PD85 & 385 & 19 & 1 \\
PD89 & 315 & 16 & 5 \\
\hline
\end{tabular}

${ }^{a}$ Data were derived from Table 4 . Classes 1 and 4 represent transfer of the nontranscribed strand, and classes 2 and 3 represent transfer of the transcribed strand.

mutation (the same single-base-pair insertion used in our study). Although the frecuency of PMS for the arg4-16 mutation observed by Williamson et al. (32) appears lower than we observed, in other studies of the arg4-16 mutation by the same researchers, a PMS frequency of $48 \%$ was reported (8). In addition, Kramer et al. (13) observed PMS frequencies/aberrant segregation for various combinations of mismatches at the $A D E 8$ locus with the following results: A/A-T/T, 8\%; G/T-C/A, 3\%; G/A-T/C, 6\%; and C/C-G/G, $37 \%$. Thus, our data concerning the frequency of PMS for different mismatches are in good quantitative and qualitative agreement with that of the Fogel laboratory.

Nicolas et al. (21) and Lichten et al. (15) examined PMS for a G-to-C mutation in the ATG initiating codon of $A R G 4$ (arg4-nsp). These workers observed that PMS events comprised about $13 \%$ of the total aberrant tetrads, a frequency significantly lower than that observed in either our study or that of the Fogel group $(7,8)$. Several explanations for this difference are possible. First, the strains used by Lichten et al. may be more repair proficient than those used by the other groups. Second, the efficiency of repair may be affected by neighboring sequences. Third, a substantial fraction of the gene conversions of arg4-nsp do not involve mismatch repair of a heteroduplex. It has been suggested that some conversion events involve repair of a doublestranded gap (30). This possibility is made unlikely by the observation that the pmsl mutation, which reduces the efficiency of mismatch repair (32), increases the frequency of PMS of arg4-nsp to about $50 \%$ (15). Fourth, the strains used by Lichten et al. (15) may include an additional mismatch that affects repair of the arg4-nsp mismatch, although precautions were taken to avoid extraneous mismatches (14a). Since well-repaired mismatches increase the repair frequency of nearby poorly repaired mismatches in yeast cells

TABLE 7. Comparison of the spectra of aberrant segregations at HIS4 for conventional tetrad dissection and dissection altered to monitor spore phenotype

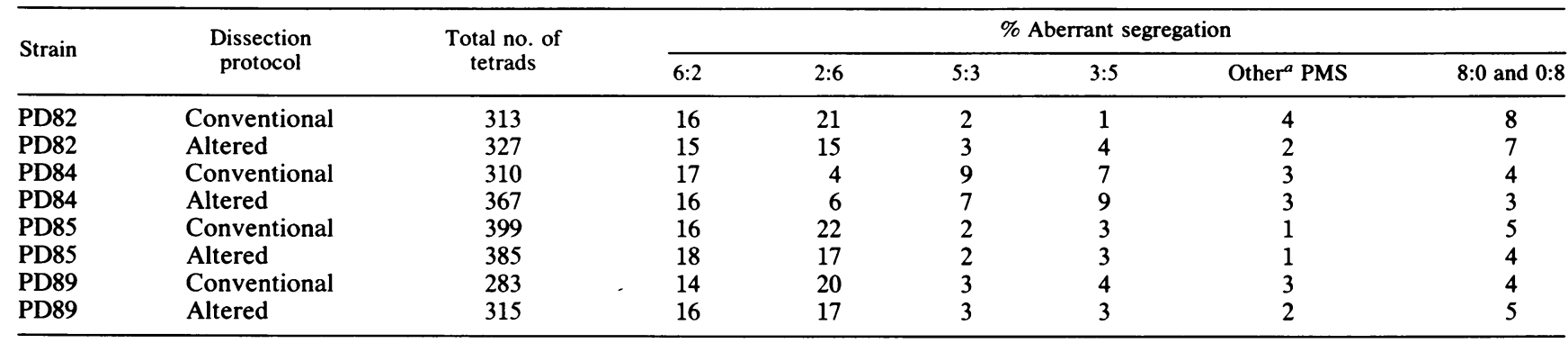

\footnotetext{
${ }^{a}$ Includes tetrads with two or more sectors.
} 
$(7,10)$, such mismatches would be expected to lower the PMS frequency.

In agreement with Lichten et al. (15), we find that the meiotic repair of the $\mathrm{C} / \mathrm{C}$ mismatch is less efficient than repair of the $\mathrm{G} / \mathrm{G}$ mismatch; unrepaired mismatches of both types, however, are detected. In the former study, no heteroduplexes containing G/G mismatches were observed in PMS1 strains, and the authors concluded that there was at least a fivefold difference in the number of $C / C$ and $G / G$ unrepaired mismatches. The relative frequencies of unrepaired $\mathrm{C} / \mathrm{C}$ and $\mathrm{G} / \mathrm{G}$ mismatches are significantly different in the two studies (chi-square value of 5.3).

For the mutations at $A R G 4$ that resulted in a C/C-G/G mismatch $(7,15,21)$, the $6: 2$ class exceeded the $2: 6$ class (by 1.7-fold) and the 3:5 class exceeded the 5:3 class (by 3-fold). These patterns are expected if the nontranscribed strand is usually the donor strand and $\mathrm{C} / \mathrm{C}$ mismatches are more poorly repaired than $\mathrm{G} / \mathrm{G}$ mismatches (Fig. 1). In our experiments, the frequency of 5:3 and 3:5 tetrads is approximately the same, and the frequency of $6: 2$ tetrads exceeds that of $2: 6$ tetrads by threefold. One explanation of the difference between the behavior of the his 4 and arg 4 mutations in the $5: 3$ and $3: 5$ classes is that a significant fraction of the $G / G$ mismatches in HIS4 (but not ARG4 [15]) remain unrepaired. These unrepaired mismatches would reduce the expected difference between the 5:3 and 3:5 classes.

The mitotic repair of different types of mismatches was examined in two studies in which heteroduplexes constructed in vitro were transformed in vegetatively dividing cells $(1,13)$. Since the mismatches were within a gene that affected cell color, failure to repair the mismatch was indicated by color-sectored colonies derived from single transformed cells. In one study (13), the $\mathrm{C} / \mathrm{C}$ mismatches were poorly repaired relative to all other mismatches, whereas in the other study (1), A/A, T/T, and $\mathrm{C} / \mathrm{C}$ mismatches were inefficiently repaired (to approximately the same degree) relative to the other mismatches. The close resemblance of our results and those of Kramer et al. (13) suggests that the mitotic and meiotic mismatch repair systems are either identical or very similar.

In Escherichia coli and Streptococcus pneumoniae, C/C, $\mathrm{G} / \mathrm{A}$, and T/C mismatches are generally inefficiently corrected relative to the other types of point mismatches, although efficient repair of $G / A$ and $T / C$ mismatches has been observed in some sequence contexts (reviewed in reference 17). It is possible that the mismatch repair evolved to remove errors caused by $\mathrm{DNA}$ polymerase. If $\mathrm{C} / \mathrm{C}$ mismatches caused by such errors are very rare, there may be no selective advantage to having a mismatch repair system that efficiently repairs such mismatches (12). Alternatively, the inefficient repair of $\mathrm{C} / \mathrm{C}$ may be a consequence of enzymatic systems in yeast and $E$. coli cells that recognize only specific types of helical distortions (17).

Direction of mismatch correction: the ratio of conversion to restoration. The level of PMS for a particular mutation is a function of only two variables, the frequency of heteroduplex formation involving that mutation and the efficiency with which the resulting mismatch is repaired. The frequency of gene conversion involves a third variable, the direction with which the mismatch is corrected. As described above, if a wild-type strand invades a mutant gene and the resulting mismatch is repaired to generate a wildtype gene, the tetrad would have a 6:2 gene conversion event. Repair of the mismatch to generate a mutant gene (restoration event) would lead to 4:4 segregation. Thus, the ratio of conversion to restoration, at least in principle, can greatly affect the observed level of gene conversion. Below, we address two issues. First, we discuss the possibility that, for mutations at the $5^{\prime}$ end of $H I S 4$, conversion is more common than restoration. Second, we discuss the evidence that the C/C-G/G mismatch responds differently than the other mismatches to the system that controls the ratio of conversion and restoration.

Conversion-type repair exceeds restoration-type repair för well-repaired mutant alleles at the $5^{\prime}$ end of HIS4. The ratio of conversion to restoration is not known for any mutation in $S$. cerevisiae. From analysis of gene conversion and crossing over at the HISI locus in yeast cells, Hastings (9) concluded that conversion-type repair was more common than restoration-type repair. This analysis required the assumption that crossovers occur only at the low-conversion end of the gene. Since studies with other yeast genes (7) indicate that crossovers can occur at either the high- or low-conversion end of the gene, the relative amounts of conversion and restoration are not unambiguously determined by these experiments.

Another argument that conversion may exceed restoration is based on the frequencies of aberrant segregations of alleles that are close together within a gene but have very different amounts of PMS. For example, an insertion of a small palindromic nucleotide about $50 \mathrm{bp}$ upstream of the +1 ATG of HIS4 (his4-B2) had a frequency of 5:3 and 3:5 tetrads of $24 \%\left(U_{H}\right.$ [frequency of unrepaired heteroduplexes for highPMS allele] $=24 \%$ ) of the total tetrads and a level of $6: 2$ and $2: 6$ tetrads of $15 \%\left(\mathrm{C}_{\mathbf{H}}\right.$ [frequency of conversion tetrads for high-PMS allele] $=15 \%$ ) (20). For mutations in the ATG sequence from this study (excluding the his4-ATC mutation), the average frequency (of total tetrads) of 5:3 and 3:5 tetrads was $5 \%\left(\mathrm{U}_{\mathrm{L}}=5 \%\right)$ with an average conversion frequency (6:2 and $2: 6$ tetrads) of $35 \%\left(C_{L}=35 \%\right)$. Since $24 \%$ of the tetrads in the high-PMS strain and $5 \%$ of tetrads in the low-PMS strain represent PMS events, $19 \%\left(U_{H}-U_{L}\right)$ of the tetrads in the low-PMS strain presumably represent mismatch repair events in excess of those observed in the high-PMS strain. If the excess repair events are distributed equally between restoration- and conversion-type repair, then about $25 \%\left[\left(U_{H}-U_{L}\right) / 2+C_{H}\right]$ of the tetrads in the low-PMS strains should represent gene conversion events. If these repair events are all of the conversion type, approximately $34 \%\left[\left(U_{H}-U_{L}\right)+C_{H}\right]$ of the tetrads should represent gene conversion events, which is close to the observed value (35\%). The conclusion that most mismatch repair is conversion-type repair is, however, not completely rigorous, since it is not clear that the level of heteroduplex formation is the same for mutations at the same position within a gene.

There are several plausible methods to ensure that conversion-type repair exceeds restoration-type repair. First, the yeast cell may tag the donor strand in such a way that this strand is used as a template for repair of the mismatch or tag the recipient strand to prevent it from being used as a template. Alternatively, the yeast cell may tag the nontranscribed strand at the HIS4 locus such in a way that this strand is used as a template for repair (or tag the transcribed strand to prevent it from being used as a template); since the nontranscribed strand is preferentially donated, this mechanism would result in an excess of conversion-type relative to restoration-type repair. All of these mechanisms are analogous to the major mismatch repair system observed in $E$. coli, in which a strand tagged by methylation is protected from mismatch repair relative to an unmethylated strand (17, $24)$. In the methyl-directed repair system in $E$. coli, the efficiency of repair in hemimethylated DNA is primarily a 
function of the specific mismatch, with the $\mathrm{C} / \mathrm{C}$ mismatch being repaired least efficiently (as discussed previously).

In summary, base substitutions at the 5' end of HIS4 that lead to well-repaired mismatches have the frequency of gene conversion expected if conversion-type repair exceeds restoration-type repair. The ratio of conversion to restoration appears to be constant for the well-repaired mismatches at the 5' end of HIS4, since the frequency of 6:2 and 2:6 tetrads is approximately equal and a difference in the conversion/restoration ratio would lead to disparity in the frequency of 6:2 and 2:6 tetrads. Although the ratio of conversion to restoration is a constant for a particular position within a gene, it is possible that the ratio differs at different positions. At HIS4, as well as for several other yeast genes (reviewed in reference 23), the frequency of gene conversion is higher at one end of the gene than at the other. Such gradients are usually interpreted as indicating that heteroduplex formation initiates near one end of the gene and the heteroduplex is propagated toward the other end of the gene $(10,11)$. An alternative possibility is that the polarity gradient reflects an alteration in the ratio of conversion- and restoration-type repair along the gene. If this ratio is affected by a signal or tag located near one end of the gene that is propagated in a distance-dependent way toward the other end of the gene, a polarity gradient would be formed. This possibility could be checked by examining the frequency of segregation of high-PMS alleles near the $3^{\prime}$ end of HIS4. If the polarity gradient reflects mismatch repair, such mutant alleles should show less of a polarity gradient than wellrepaired alleles.

Conversion and restoration repair for the $\mathrm{C} / \mathrm{C}-\mathrm{G} / \mathrm{G}$ mismatch. As discussed above, the equal frequency of $6: 2$ and 2:6 tetrads for the well-repaired mismatches at the $5^{\prime}$ end of HIS4 indicates that levels of conversion-type and restoration-type repair are the same for all of these mismatches. We argue below that the inequality of $6: 2$ and 2:6 tetrads for strains containing the $\mathrm{C} / \mathrm{C}-\mathrm{G} / \mathrm{G}$ mismatch is likely to be the result of a difference in the ratio of conversion to restoration for the $\mathrm{C} / \mathrm{C}$ mismatch relative to the $\mathrm{G} / \mathrm{G}$ mismatch. The three variables influencing the frequency of conversion are the frequency of heteroduplex formation, the efficiency of repair, and the direction of repair. Since for all mutations leading to a single-base-pair mismatch (except the mutation resulting in the $\mathrm{C} / \mathrm{C}-\mathrm{G} / \mathrm{G}$ mismatch) the number of $6: 2$ and 2:6 tetrads is very similar (Table 2), it is likely that the wild-type and mutant genes initiate heteroduplex formation with the same frequency. In addition, the disparity in gene conversion of the his4-ATC mutation cannot be the result of one chromosome donating strands more frequently than the other, since the same disparity is observed for both PD84 and JS102, which have the mutant his 4 allele on opposite homologs (Table 2). Since the level of 5:3 and 3:5 tetrads in these strains in PD84 and JS102 is the same, the mismatches resulting from the transfer of a wild-type strand appear to be repaired at a similar frequency to those resulting from transfer of a mutant strand. Thus, the only variable that is likely to explain the disparity in gene conversion in PD84 and JS102 is the relative amounts of conversion and restoration.

A number of related mechanisms could produce the observed effects on gene conversion. For example, if all of the $\mathrm{G} / \mathrm{G}$ mismatches are corrected by conversion-type repair and all of the $\mathrm{C} / \mathrm{C}$ mismatches corrected by restoration-type repair, an excess of 6:2 tetrads would be observed. Alternatively, if the nontranscribed strand always acts as a template for repair of the $\mathrm{G} / \mathrm{G}$ mismatches (leading to conversion-type repair when the wild-type strand is donated and restoration- type repair when the mutant strand is donated), and if the $\mathrm{C} / \mathrm{C}$ mismatch is converted and restored with equal frequency (regardless of which strand is donated), an excess of 6:2 tetrads would result. Both of these models suggest the possibility that the repair of $\mathrm{C} / \mathrm{C}$ mismatches is different from the repair of other mismatches in two ways: the frequency of repair and the type of repair (the conversion-restoration ratio). It should be noted that this effect may be dependent on the context of the $\mathrm{C} / \mathrm{C}-\mathrm{G} / \mathrm{G}$ mismatch, since for the same mismatch at the $A R G 4$ locus, the level of $6: 2$ and $2: 6$ tetrads was less different $(7,15,21)$.

The nontranscribed strand is the favored donor in heteroduplexes with an unrepaired mismatch at HIS4. In our study, as in previous studies $(15,19)$, unrepaired mismatches were more frequently observed in tetrads in which the nontranscribed strand was a donor than in those in which the transcribed strand was a donor. This observation has two possible interpretations. Our favored interpretation is that the initiation of strand transfer preferentially involves the nontranscribed strand as a donor (19). The alternative possibility is that strands are transferred with equal frequencies, but heteroduplexes in which the transcribed strand is a donor are more efficiently repaired. As discussed previously, the twofold bias in favor of transfer of the nontranscribed strand observed with the his4-ATC mutation is significantly less than observed with some of the other mutations. This result suggests the possibility that $\mathrm{G} / \mathrm{G}$ and $\mathrm{C} / \mathrm{C}$ mismatches may be repaired with different efficiencies, depending on which strand (transcribed or nontranscribed) is used as a donor for heteroduplex formation. A unifying hypothesis for these and other observations is that the rules governing repair of $\mathrm{C} / \mathrm{C}$ mismatches are different in three ways from those influencing the repair of other point mismatches. First, the $\mathrm{C} / \mathrm{C}$ mismatch appears to be repaired less efficiently. Second, the repair events that do occur may involve a different ratio of conversion to restoration than for other mismatches at the same position in the gene. Third, the efficiency of repair of the $\mathrm{C} / \mathrm{C}$ mismatch may be affected by the strand that is transferred to form the heteroduplex.

\section{ACKNOWLEDGMENTS}

We thank T. F. Donahue, A. M. Cigan, B. Ruskin, F. Dietrich, and G. R. Fink for plasmids used in this study and members of the Petes laboratory for comments on the manuscript. We thank Margaret Dominska for expert technical assistance and Gail Tudor for advice on the statistical analysis.

This research was supported by NIH grant 24110 and by an NIH training grant at the University of Chicago (P.D.).

\section{REFERENCES}

1. Bishop, D. K., J. Anderson, and R. D. Kolodner. 1989. Specificity of mismatch repair following transformation of Saccharomyces cerevisiae with heteroduplex plasmid DNA. Proc. Natl. Acad. Sci. USA 86:3713-3717.

2. Bishop, D. K., and R. D. Kolodner. 1986. Repair of heteroduplex plasmid DNA after transformation into Saccharomyces cerevisiae. Mol. Cell. Biol. 6:3401-3409.

3. Boeke, J. D., F. Lacroute, and G. R. Fink. 1984. A positive selection for mutants lacking orotidine-5'-phosphate decarboxylase activity in yeast: 5-fluoro-orotic acid resistance. Mol. Gen. Genet. 197:345-346.

4. Dohet, C., R. Wagner, and M. Radman. 1985. Repair of defined single base pair mismatches in E. coli. Proc. Natl. Acad. Sci. USA 82:503-505.

5. Donahue, T. F., and A. M. Cigan. 1988. Genetic selection for mutations that reduce or abolish ribosomal recognition of the HIS4 translational initiator region. Mol. Cell. Biol. 8:2955-2963. 6. Donahue, T. F., P. J. Farabaugh, and G. R. Fink. 1982. The 
nucleotide sequence of the HIS4 region of yeast. Gene 18:47-59.

7. Fogel, S., R. K. Mortimer, and K. Lusnak. 1981. Mechanisms of meiotic gene conversion, or "wanderings on a foreign strand," p. 289-339. In J. Strathern, E. W. Jones, and J. Broach (ed.), The molecular biology of the yeast Saccharomyces, vol. I. Cold Spring Harbor Laboratory, Cold Spring Harbor, N.Y.

8. Fogel, S., R. K. Mortimer, K. Lusnak, and F. Tavares. 1979. Meiotic gene conversion-a signal of the basic recombination events in yeast. Cold Spring Harbor Symp. Quant. Biol. 43: $1325-1341$.

9. Hastings, P. J. 1984. Measurement of restoration and conversion: its meaning for the mismatch repair hypothesis of conversion. Cold Spring Harbor Symp. Quant. Biol. 49:49-53.

10. Hastings, P. J. 1987. Meiotic recombination interpreted as heteroduplex correction, p. 107-137. In P. B. Moens (ed.), Meiosis. Academic Press, Inc., New York.

11. Holliday, R. 1964. A mechanism for gene conversion in fungi. Genet. Res. 5:282-304.

12. Kramer, B., W. Kramer, and H. Fritz. 1984. Different base/base mismatches are corrected with different efficiencies by the methyl-directed DNA mismatch-repair system of $E$. coli. Cell 38:879-887.

13. Kramer, B., W. Kramer, M. S. Williamson, and S. Fogel. 1989. Heteroduplex DNA correction in yeast is mismatch specific and requires functional PMS genes. Mol. Cell. Biol. 9:4432-4440.

14. Kramer, W., B. Kramer, M. S. Williamson, and S. Fogel. 1989. Cloning and nucleotide sequence of DNA mismatch repair gene PMSI from Saccharomyces cerevisiae: homology to procaryotic $m u t L$ and hexB. J. Bacteriol. 171:5339-5346.

14a.Lichten, M. Personal communication.

15. Lichten, M., C. Goyon, N. P. Schultes, D. Treco, J. W. Szostak, J. E. Haber, and A. Nicolas. 1990. Detection of heteroduplex DNA molecules among the products of Saccharomyces cerevisiae meiosis. Proc. Natl. Acad. Sci. USA 87:7653-7657.

16. Meselson, M., and C. Radding. 1975. A general model for genetic recombination. Proc. Natl. Acad. Sci. USA 72:358-361.

17. Modrich, P. 1987. DNA mismatch correction. Annu. Rev. Biochem. 56:435-466.

18. Moore, C. W., D. M. Hampsey, J. F. Ernst, and F. Sherman. 1988. Differential mismatch repair can explain the disproportionalities between physical distances and recombination frequencies of $c y c l$ mutations in yeast. Genetics 119:21-34.

19. Nag, D. K., and T. D. Petes. 1990. Genetic evidence for preferential strand transfer during meiotic recombination in yeast. Genetics 125:753-761.

20. Nag, D. K., M. A. White, and T. D. Petes. 1989. Palindromic sequences in heteroduplex DNA inhibit mismatch repair in yeast. Nature (London) 340:318-320.

21. Nicolas, A., D. Treco, N. P. Shultes, and J. W. Szostak. 1989. An initiation site for gene conversion in the yeast Saccharomyces cerevisiae. Nature (London) 338:35-39.

22. Orr-Weaver, T. L., J. W. Szostak, and R. Rothstein. 1981. Yeast transformation: a model system for the study of recombination. Proc. Natl. Acad. Sci. USA 78:6354-6358.

23. Petes, T. D., R. E. Malone, and L. S. Symington. In J. Broach, E. Jones, and J. Pringle (ed.), The molecular and cellular biology of the yeast Saccharomyces, in press. Cold Spring Harbor Laboratory, Cold Spring Harbor, N.Y.

24. Pukkila, P. J., J. Peterson, G. Herman, P. Modrich, and M. Meselson. 1983. Effects of high levels of DNA adenine methylation on methyl-directed repair in Escherichia coli. Genetics 104:571-582.

25. Radding, C. M. 1982. Homologous pairing and strand exchange in genetic recombination. Annu. Rev. Genet. 16:405-437.

26. Radman, M., and R. Wagner. 1986. Mismatch repair in Escherichia coli. Annu. Rev. Genet. 20:523-538.

27. Scherer, S., and R. W. Davis. 1979. Replacement of chromosome segments with altered DNA sequences constructed in vitro. Proc. Natl. Acad. Sci. USA 76:4951-4955.

28. Sherman, F., G. R. Fink, and J. B. Hicks. 1982. Methods in yeast genetics. Cold Spring Harbor Laboratory, Cold Spring Harbor, N.Y.

29. Symington, L. S., and T. D. Petes. 1988. Expansions and contractions of the genetic map relative to the physical map of yeast chromosome III. Mol. Cell. Biol. 8:595-604.

30. Szostak, J. W., T. L. Orr-Weaver, R. J. Rothstein, and F. W. Stahl. 1983. The double-strand-break repair model for recombination. Cell 33:25-35.

31. White, J. H., K. Lusnak, and S. Fogel. 1985. Mismatch-specific post-meiotic segregation frequency in yeast suggests a heteroduplex recombination intermediate. Nature (London) 315:350352.

31a.White, M., P. Detlof, M. Strand, and T. Petes. Unpublished data.

32. Williamson, M. S., J. C. Game, and S. Fogel. 1985. Meiotic gene conversion mutants in Saccharomyces cerevisiae. I. Isolation and characterization of $p m s l-1$ and pmsl-2. Genetics 110:609646.

33. Winston, F., F. Chumley, and G. R. Fink. 1983. Eviction and transplacement of mutant genes in yeast. Methods Enzymol. 101:211-228. 